\title{
GMOSS: All-sky model of spectral radio brightness based on physical components and associated radiative processes
}

DOI:

10.3847/1538-3881/153/1/26

\section{Document Version}

Final published version

Link to publication record in Manchester Research Explorer

Citation for published version (APA):

Rao, M. S., Subrahmanyan, R., Shankar, N. U., \& Chluba, J. (2016). GMOSS: All-sky model of spectral radio brightness based on physical components and associated radiative processes. The Astronomical Journal, 153(1). https://doi.org/10.3847/1538-3881/153/1/26

\section{Published in:}

The Astronomical Journal

\section{Citing this paper}

Please note that where the full-text provided on Manchester Research Explorer is the Author Accepted Manuscript or Proof version this may differ from the final Published version. If citing, it is advised that you check and use the publisher's definitive version.

\section{General rights}

Copyright and moral rights for the publications made accessible in the Research Explorer are retained by the authors and/or other copyright owners and it is a condition of accessing publications that users recognise and abide by the legal requirements associated with these rights.

\section{Takedown policy}

If you believe that this document breaches copyright please refer to the University of Manchester's Takedown Procedures [http://man.ac.uk/04Y6Bo] or contact uml.scholarlycommunications@manchester.ac.uk providing relevant details, so we can investigate your claim.

\section{OPEN ACCESS}




\title{
GMOSS: ALL-SKY MODEL OF SPECTRAL RADIO BRIGHTNESS BASED ON PHYSICAL COMPONENTS AND ASSOCIATED RADIATIVE PROCESSES
}

\author{
Mayuri Sathyanarayana Rao ${ }^{1,2}$, Ravi Subrahmanyan ${ }^{1}$, N Udaya Shankar ${ }^{1}$, and Jens Chluba ${ }^{3}$ \\ ${ }^{1}$ Raman Research Institute, C V Raman Avenue, Sadashivanagar, Bangalore 560080, India; mayuris@ $@$ rri.res.in \\ ${ }^{2}$ Australian National University, Research School for Astronomy \& Astrophysics, Mount Stromlo Observatory, Cotter Road, Weston, ACT 2611, Australia \\ 3 Jodrell Bank Centre for Astrophysics, University of Manchester, Oxford Road, M13 9PL, UK \\ Received 2016 July 24; revised 2016 October 9; accepted 2016 October 11; published 2016 December 22
}

\begin{abstract}
We present the Global Model for the Radio Sky Spectrum (GMOSS), a novel, physically motivated model of the low-frequency radio sky from $22 \mathrm{MHz}$ to $23 \mathrm{GHz}$. GMOSS invokes different physical components and associated radiative processes to describe the sky spectrum over 3072 pixels of $5^{\circ}$ resolution. The spectra are allowed to be convex, concave, or of more complex form with contributions from synchrotron emission, thermal emission, and free-free absorption included. Physical parameters that describe the model are optimized to best fit four all-sky maps at $150 \mathrm{MHz}, 408 \mathrm{MHz}, 1420 \mathrm{MHz}$, and $23 \mathrm{GHz}$ and two maps at 22 and $45 \mathrm{MHz}$ generated using the Global Sky Model of de Oliveira-Costa et al. The fractional deviation of the model from data has a median value of $6 \%$ and is less than $17 \%$ for $99 \%$ of the pixels. Though aimed at the modeling of foregrounds for the global signal arising from the redshifted $21 \mathrm{~cm}$ line of hydrogen during the Cosmic Dawn and the Epoch of Reionization (EoR), over redshifts $150 \lesssim z \lesssim 6$, GMOSS is well suited for any application that requires simulating spectra of the lowfrequency radio sky as would be observed by the beam of any instrument. The complexity in spectral structure that naturally arises from the underlying physics of the model provides a useful expectation for departures from smoothness in EoR foreground spectra and hence may guide the development of algorithms for EoR signal detection. This aspect is further explored in a subsequent paper.
\end{abstract}

Key words: cosmic background radiation - cosmology: observations - ISM: general - methods: data analysis methods: observational - radio continuum: general

\section{INTRODUCTION}

Interest in the low-frequency radio sky goes back to the very early days of radio astronomy. Early measurements at $20.5 \mathrm{MHz}$ by Karl Jansky showed that the Galaxy itself is a strong emitter of radiation at low frequencies. The source of such Galactic emission was poorly understood and was attributed to a wide variety of phenomena, right from radio stars to dust grains. It is now understood that the predominant radiative mechanism contributing to this Galactic emission at long wavelengths is synchrotron radiation from relativistic electrons spiraling around Galactic magnetic field lines. With many experiments currently underway attempting to detect redshifted $21 \mathrm{~cm}$ spectral signatures arising from the Cosmic Dawn and the Epoch of Reionization (EoR), there is renewed interest in understanding the precise spectral shapes of the lowfrequency radio sky, which forms a strong foreground to the weak cosmological signal.

The radio sky is composed of signals from our Galaxy, extragalactic radio sources, and the cosmic microwave background (CMB), with added spectral distortions related to the cosmic thermal history of baryons, structure formation, energy release in the early universe, and interactions between propagating radiation and gas. These include distortions from the Cosmic Dawn and EoR. The emergence of the first sources in the Cosmic Dawn and the transition of baryonic matter in the universe from being almost completely neutral to its present mostly ionized form during EoR is an interesting and poorly constrained period in cosmology. During these times, the cosmological evolution of the spin temperature of hydrogen and reionization as a result of first light from the first collapsed objects is expected to leave an imprint as redshifted $21 \mathrm{~cm}$ emission and absorption (see Madau et al. 1997; Shaver et al. 1999). These $21-\mathrm{cm}$ spectral distortions are a probe of the thermal history of the gas and also of the sources and timing of reionization (Glover et al. 2014) in the redshift range of 6 to about 150. For a comprehensive review of the subject, see Furlanetto et al. (2006). There are global, all-sky isotropic spectral features as well as angular variations in spectral structure, embedded as tiny additive components in the radio background at frequencies $\lesssim 200 \mathrm{MHz}$. Radio emission from Galactic and extragalactic sources forms strong foregrounds to the cosmological signal and are orders of magnitude brighter.

It is necessary to have a realistic expectation for the radio foreground that would be observed by EoR detection experiments (see Bowman et al. 2008; Patra et al. 2013; Pober et al. 2014, 2015; Voytek et al. 2014; Ali et al. 2015; Bernardi et al. 2015). Although the sky spectrum as measured by individual experiments will be instrument-specific, a generic model representative of the spectral distribution of intensities in the low-frequency radio sky and a method to simulate the expected contribution of the same to spectra observed in EoR detection telescopes will be a powerful tool in formulating data analysis methods.

Here we present a physically motivated model of the lowfrequency radio sky: the Global Model for the Radio Sky Spectrum (GMOSS). GMOSS is a generic model and can be used to generate spectra of the low-frequency radio sky for other applications as well. One such application is to simulate the expected foregrounds for other spectral distortions of the $\mathrm{CMB}$, such as those arising from the Epoch of Recombination (Sunyaev \& Chluba 2009). As noted in Sathyanarayana Rao et al. (2015), the optimal frequency for a ground-based detection of cosmological recombination lines is an octave band in the range of $2-6 \mathrm{GHz}$. Over these frequencies the 
recombination signal is expected to have a quasi-periodic sinusoidal shape, whereas the foregrounds are expected to be smooth. However, since the cosmological recombination signal is at least eight orders of magnitude weaker than the foreground, a thorough treatment of the expectation of the spectral shapes inherent in the foreground can be provided by GMOSS.

The motivation for GMOSS in comparison to existing sky models is given in Section 2. GMOSS itself is described in Section 3 , and a discussion on the distribution of parameters and the goodness of fit is presented in Section 4.

\section{MOTIVATION}

Precise measurement of foreground spectra is a goal of experiments aiming to detect redshifted $21 \mathrm{~cm}$ signatures from the Cosmic Dawn and EoR (hereafter referred to in totality as EoR). Disentangling spectral structure specific to the EoR signal from those of the foreground (Harker 2015) and instrument (Switzer \& Liu 2014) is a challenge. In generic models (such as Figure 1 of Pritchard \& Loeb 2010a), the redshifted $21 \mathrm{~cm}$ signal (in the $10-200 \mathrm{MHz}$ window) is expected to show multiple turning points arising from various physical processes, such as X-ray and UV heating, ionization of the gas, and Wouthuysen-Field coupling of spin to kinetic temperature via $\operatorname{Ly} \alpha$, to name a few (for a description of the physics that determine the turning points in the EoR signal, see Pritchard \& Loeb 2010b). On the other hand, it is assumed that over the same frequency range, foregrounds are smooth (see Petrovic \& Oh 2011) and that "smoothness" is captured by low-order polynomials in log brightness temperature versus log frequency space (we hereinafter refer to this domain as log-log space). It has been proposed to exploit this supposed smoothness of foregrounds to distinguish them from the global EoR signal, which in the generic form is expected to show multiple turning points between 10 and $200 \mathrm{MHz}$. However, it is uncertain if this assumption of an inherently smooth foreground is indeed correct. For instance, a mechanism that might result in inflections of the observed spectrum is the combined emission from steep and flat spectrum sources along with radiation from sources that have a break in the electron energy distribution. Furthermore, flattening of this combined spectrum due to absorption at low frequencies by a thermal interstellar medium along the line of sight and at high frequencies by free-free emission can introduce additional shapes in the spectrum. Though the assumption is that the averaging of multiple spectra of various shapes across the sky and along the line of sight must result in an observed spectrum that is devoid of sharp spectral features, the underlying spectral energy distribution that results in the radiation would ultimately determine the inherent smoothness of the observed spectrum. An interpolating function that is guided by physical processes provides a non-trivial treatment of the shape of the foreground spectrum.

Waelkens et al. (2009) present a tool to simulate maps of the total and polarized synchrotron emission of the radio sky, including effects of Faraday rotation. Other popular sky models, such as the Global Sky Model (GSM; de OliveiraCosta et al. 2008; with recent improvements made by Zheng et al. 2016), use data-driven methods to generate snapshots of the sky at frequencies between those where large-area maps are available. This is useful in creating all-sky maps at discrete frequencies and may also be used to generate spectra in any sky direction by computing the sky brightness over a contiguous range of frequencies. It is estimated that for sub-GHz frequencies predicted maps from the GSM are at worst in error by $\lesssim 10 \%$ depending on the region of the sky. To date, in the literature, mock spectra have been generated by first computing the beam-weighted temperatures from maps at discrete frequencies and then interpolating with either power laws or low-order polynomials to simulate EoR foregrounds. For instance, Pritchard \& Loeb (2010b) generate a mock spectrum by allowing the GSM-generated sky to drift over the zenith of an ideal frequency-independent $\cos ^{2}$ antenna beam for $24 \mathrm{hr}$. They find that their mock spectra can be fit to $\mathrm{mK}$ precision with a cubic polynomial in log-log space. Independently, Bernardi et al. (2015) find that to fit the spectrum of synchrotron emission from mono-energetic cosmic ray electrons to within $100 \mathrm{mK}$ maximum errors, a polynomial of the sixth order is required in $\log -\log$ space. For the case of synchrotron emission from an evolved population of cosmic ray electrons diffusing through the Galactic halo, the order of the polynomial required reduces to four. Harker et al. (2016) produce mock sky spectra that contain foregrounds generated using polynomial interpolation and fit them with polynomials of similar order to remove the foregrounds, eliminating any ambiguity arising from using polynomials of a different order. While this helps in focusing on statistical inference methods for the parameters of the cosmological signal itself, it remains to be examined what the precise shape of the foreground spectrum might be and to which extent it might confuse detection of the $21 \mathrm{~cm}$ signal from EoR.

What is required is a model of the foreground that encapsulates the underlying physics that gives rise to the foreground spectrum. Whether or not the resultant spectrum is smooth would guide the strategies employed to detect the EoR signal that is embedded in a measurement set. If polynomials are used to model the foreground spectrum, the order of the polynomial required to fit the foreground with the precision required for detection of the EoR signal should be guided by the spectral complexity determined by the underlying physical radiative processes.

In GMOSS we present a physical sky model that includes the effects of plausible radiative processes arising from different components of the radio sky. Additionally, the physical parameters used to describe GMOSS are guided by all-sky maps at different frequencies, thus enabling a check on the goodness of the model.

\section{GMOSS: A SPATIALLY RESOLVED SPECTRAL MODEL FOR THE RADIO SKY}

Previous efforts to determine the complexity of EoR foregrounds have usually simulated sky spectra by interpolating with polynomials between brightness temperatures that were first computed for large instrument beam widths (see Harker 2015). However, even if the spectra of individual sources that lie in the beam are of power-law form, the summation of many such spectra with a distribution in spectral indices across the beam and along the line of sight would result in an observed spectrum that would no longer be a power law but something spectrally more complex. Thus, there exists a need to generate a generic sky model at higher resolutions than typical single antenna beams, which may then be convolved with a large antenna beam to generate mock spectra that are qualitatively more representative of the cumulative emission. 
GMOSS provides such a sky model wherein plausible physical processes are used to estimate the sky spectrum toward each direction. Furthermore, the parameters describing the spectral shape toward each sky pixel and hence describing the sky model are constrained by existing all-sky maps.

We use 4 available all-sky maps at $150 \mathrm{MHz}$ (Landecker \& Wielebinski 1970), $408 \mathrm{MHz}$ (Haslam et al. 1982), $1420 \mathrm{MHz}$ (Reich 1982; Reich \& Reich 1986; Górski et al. 2005), and $23 \mathrm{GHz}$ (WMAP science data product ${ }^{4}$ ) to generate GMOSS. Additionally, we use all-sky maps at 22 and $45 \mathrm{MHz}$ generated using the GSM (de Oliveira-Costa et al. 2008). These last two all-sky maps are expected to closely match the raw data maps that were inputs to the GSM at the corresponding frequencies. The CMB monopole temperature, when present, was subtracted from the maps. All the maps were reduced to a common resolution of $5^{\circ}$ and represented with the "R4" nested HEALPix ${ }^{5}$ scheme in galactic coordinates. Additionally, the temperature scale of the $150 \mathrm{MHz}$ all-sky map of Landecker \& Wielebinski (1970) was corrected by subtracting an offset of $21.4 \mathrm{~K}$ and scaling the pixel intensities by a factor 1.05 to improve the accuracy of the representation (see Patra et al. 2015). An offset of $493 \mu \mathrm{K}$ was added to the $23 \mathrm{GHz}$ map to include an estimate of the uniform component missing in the differential map, as described in Sathyanarayana Rao et al. (2015). Using the resultant images, with identical beams and pixelation, we generate a physical sky model for the Galactic and Extragalactic emission (excluding the CMB) in the $22 \mathrm{MHz}-23 \mathrm{GHz}$ band, as described below.

\subsection{GMOSS: Physics}

Herein we describe GMOSS. The six maps used as inputs to generate GMOSS are shown in Figure 1.

The dominant mechanism of emission at low radio frequencies is synchrotron radiation. The total spectrum emitted by an ensemble of electrons $N(\gamma)$, with a distribution $N(\gamma) d \gamma \propto \gamma^{-\mathrm{p}} d \gamma$ and energies ranging between $\gamma_{\min }$ and $\gamma_{\max }$, is given by

$$
P_{\text {tot }}(\nu)=C \int_{\gamma_{\min }}^{\gamma_{\max }} P(\nu) \gamma^{-\mathrm{p}} d \gamma
$$

Here $\gamma$ is the Lorentz factor of the electrons, indicative of electron energy, and $P(\nu)$ is the emission spectrum from individual electrons. $p$ is the index of electron energy distribution $N(\gamma)$. We define the temperature spectral index " $\alpha$ " such that the brightness temperature $T(\nu) \propto \nu^{-\alpha}$, where $\alpha$ is related to the electron distribution index by $\alpha=\frac{p+3}{2}$. Further, $P(\nu) \propto F(x)$ with

$$
F(x)=x \int_{x}^{\infty} K_{\frac{5}{3}}(\xi) d \xi,
$$

where $x=\frac{\nu}{\nu_{\mathrm{c}}}$ and $K_{\frac{5}{3}}$ is the modified Bessel function of the second kind. $\nu_{\mathrm{c}}$ is the critical frequency given by

$$
\nu_{\mathrm{c}}=\frac{3 \gamma^{2} q B \sin \alpha}{4 \pi m c},
$$

where $B$ is the magnetic field, $\alpha$ the pitch angle between the particle (electron) velocity and magnetic field, $c$ the velocity of light in free space, $q$ the charge of the particle (electron), and $m$

\footnotetext{
WMAP Science Team.

5 http://healpix.sourceforge.net
}

its mass. We refer the reader to Rybicki \& Lightman (1986) for a detailed treatment of the synchrotron radiation process.

Spectral shapes may be described as convex, concave, or more complex. Toy models of each of the shapes, exaggerated for purposes of representation, are shown in Figure 2. In GMOSS, convex spectra are modeled as synchrotron emission arising from an ensemble of electrons having a break in their energy distribution, with steeper energy indices at higher frequencies. Concave spectra are modeled as composites of steep and flat spectrum components wherein the steep spectral component dominates at lower frequencies and the flat spectral component at higher frequencies. Spectra with more complex shapes are modeled as convex or concave with significant additional thermal absorption at low frequencies and/or optically thin free-free emission at high frequencies. The parameters that optimally describe the physical model are $C_{1}$, $\alpha_{1}, \delta_{\alpha}, \nu_{\mathrm{br}}, C_{2}, T_{\mathrm{e}}, I_{\mathrm{x}}$, and $\nu_{\mathrm{t}}$. We denote by $\alpha_{1}$ and $\alpha_{2}$ the lowand high-frequency spectral indices, respectively, for the synchrotron component of the model; parameter $\delta_{\alpha}$ is defined by the relation $\alpha_{2}=\alpha_{1}+\delta_{\alpha}$. The remaining parameters are described below, where we separately consider the modeling of pixels with synchrotron components that are convex and concave.

1. Case of pixels where $\alpha_{2}>\alpha_{1}$ : We refer to such spectra as convex. We model such spectra as primarily arising from synchrotron emission from electrons with power-law energy distributions with a break, which consequently causes the emission spectrum to be a broken power law and hence of convex form. We also allow for thermal absorption at low frequencies and optically thin thermal emission toward higher frequencies to account respectively for any low-frequency flattening and for any highfrequency excess. The functional form of the model describing the sky brightness temperature $T(\nu)$ is given by

$$
\begin{aligned}
T(\nu)= & C_{1}\left(\nu ^ { - 2 } \left\{\gamma_{\text {break }}^{2 \alpha_{1}-3} \int_{\gamma_{\min }}^{\gamma_{\text {break }}} F(x) x \gamma^{-\left(2 \alpha_{1}-3\right)} d \gamma\right.\right. \\
& \left.+\gamma_{\text {break }}^{2 \alpha_{2}-3} \int_{\gamma_{\text {break }}}^{\gamma_{\text {max }}} F(x) x \gamma^{-\left(2 \alpha_{2}-3\right)} d \gamma\right\} \\
& \left.+I_{\mathrm{x}} \nu^{-2.1}\right) e^{-\left(\frac{\nu_{\mathrm{t}}}{\nu}\right)^{2.1}}+T_{\mathrm{e}}\left(1-e^{-\left(\frac{\nu_{\mathrm{L}}}{\nu}\right)^{2.1}}\right) .
\end{aligned}
$$

The two spectral indices $\alpha_{1}$ and $\alpha_{2}=\alpha_{1}+\delta_{\alpha}\left(\delta_{\alpha}>0\right)$ are appropriately converted to electron distribution indices to derive the emission spectra. The break frequency $\nu_{\mathrm{br}}$ is related to the Lorentz factor $\gamma_{\text {break }}$ at which the energy spectrum has a break in its power-law form; the two are related by Equation (3). An optically thin thermal component with brightness parameterized by $I_{\mathrm{x}}$ is added to the synchrotron emission to account for any excess at high frequencies. The total emission is assumed to be absorbed at a turnover frequency $\nu_{\mathrm{t}}$ by a separate thermal foreground medium; this medium is assumed to have an electron temperature $T_{\mathrm{e}}$ and a constant emission measure and would also add its own emission to the sky brightness. This is captured by the terms $e^{-\left(\nu_{t} / \nu\right)^{2.1}}$ and $T_{\mathrm{e}}\left(1-e^{\left.-\left(\frac{\nu_{\mathrm{t}}}{V}\right)^{2.1}\right)}\right.$, respectively. Finally, the normalization parameter $C_{1}$ provides scaling to match the observational data in temperature units.

2. Case of pixels where $\alpha_{2}<\alpha_{1}$ : The spectra of these pixels are concave and are modeled as a composite of flat and steep spectrum synchrotron emission components with 


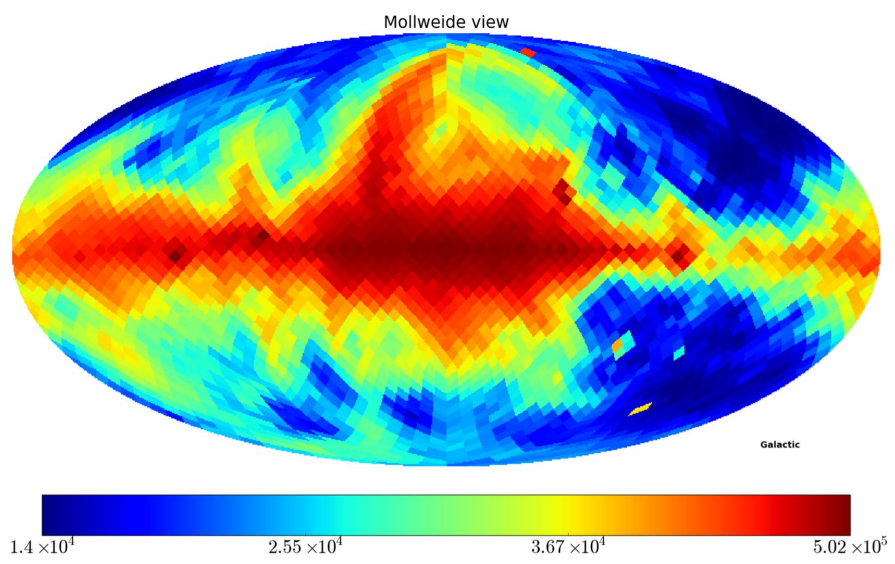

(a)

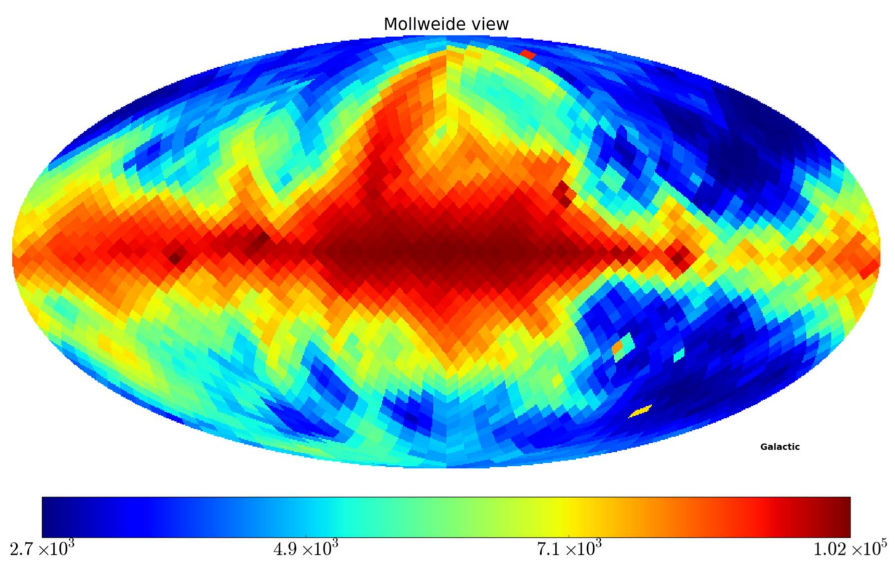

(b)

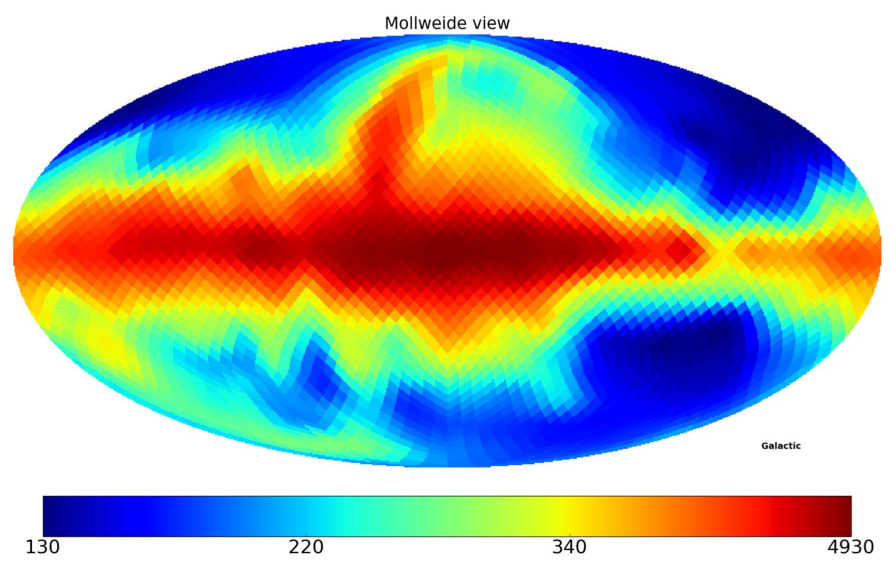

(c)

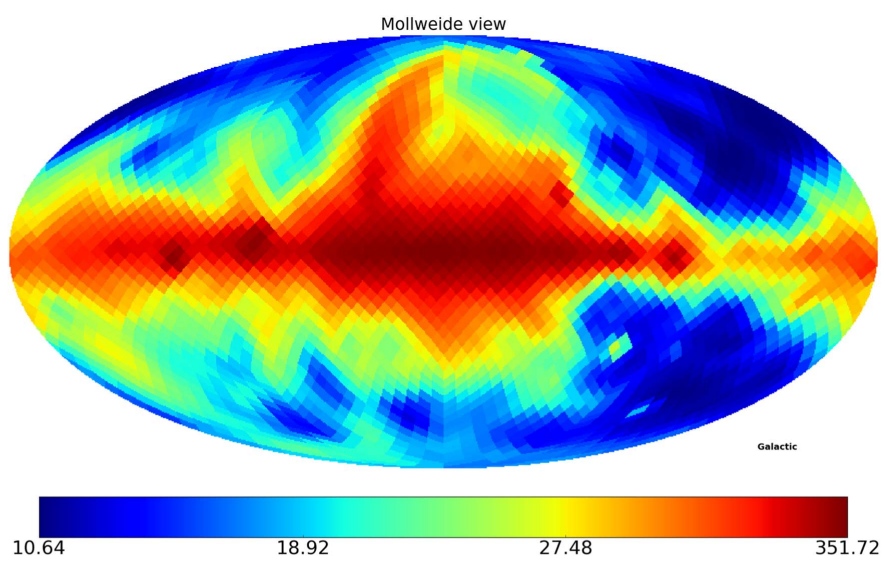

(d)

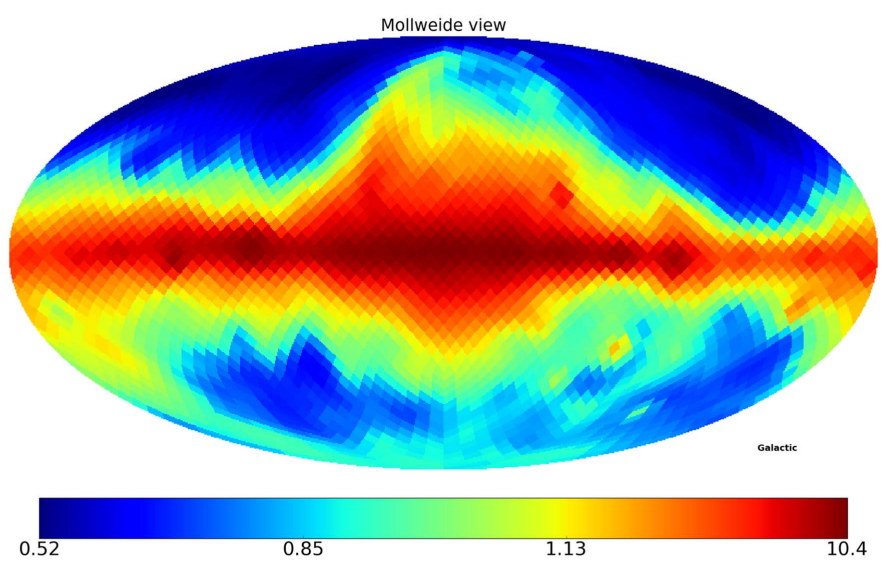

(e)

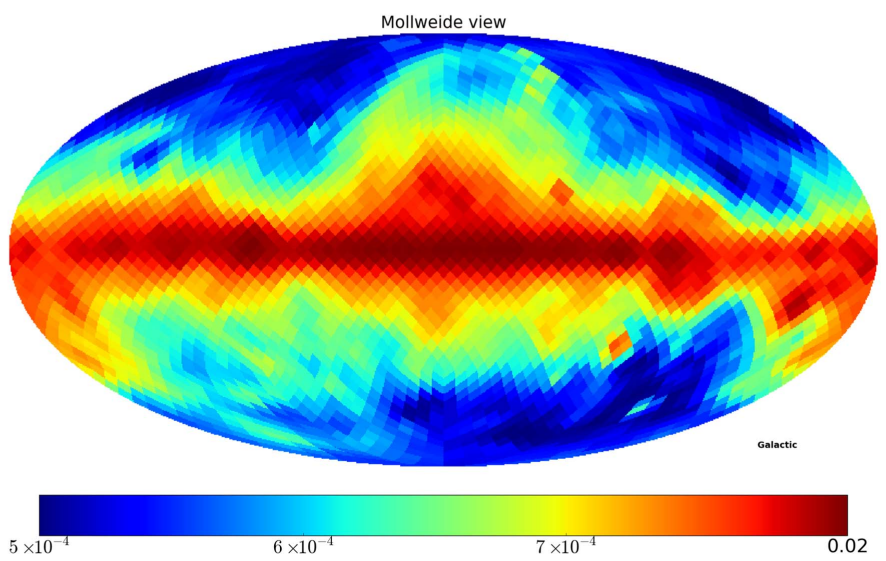

(f)

Figure 1. Maps used as input to GMOSS. Maps at (a) $22 \mathrm{MHz}$ and (b) $45 \mathrm{MHz}$ are generated from the GSM (de Oliveira-Costa et al. 2008). Map (c) is at $150 \mathrm{MHz}$ (Landecker \& Wielebinski 1970) with corrections applied from Patra et al. (2015). Maps at (d) 408 MHz (Haslam et al. 1982), (e) 1420 MHz (Reich 1982; Reich \& Reich 1986; Górski et al. 2005), and (f) $23 \mathrm{GHz}$ (WMAP science data product [WMAP Science Team]). The $23 \mathrm{GHz}$ map has an additional uniform component, as described in Sathyanarayana Rao et al. (2015). All maps are in units of kelvin at a common resolution of $5^{\circ}$ and in galactic coordinates, represented with the nested "R4" scheme of HEALPix.

individual brightness temperatures of spectral indices $\alpha_{1}$ and $\alpha_{2}=\alpha_{1}+\delta_{\alpha}\left(\delta_{\alpha}<0\right)$. Concave spectra could, in principle, be modeled as arising from concave energy distributions; however, that would be unphysical and hence not meaningful. Additionally, it is also computationally easier to model concave spectra as composed of steep and flat spectrum components than as a concave electron energy distribution. The functional form adopted 


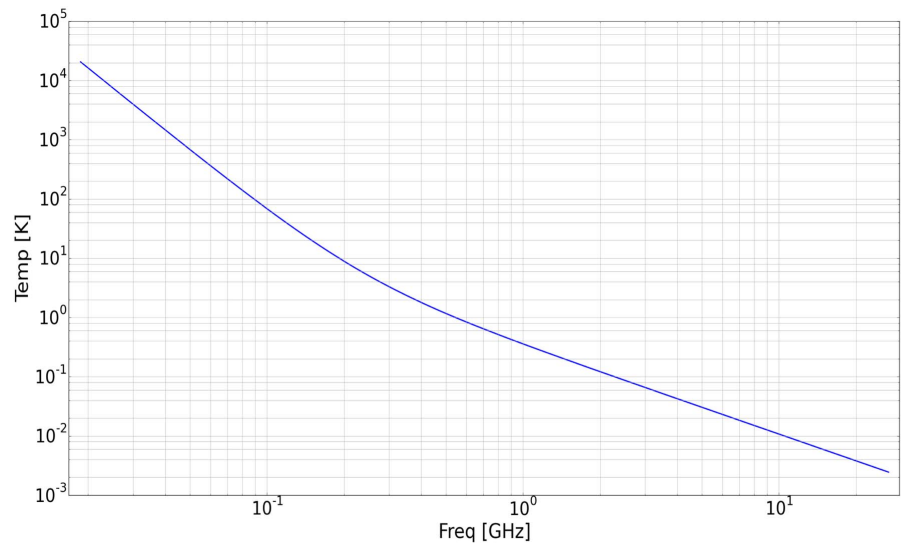

(a) Concave

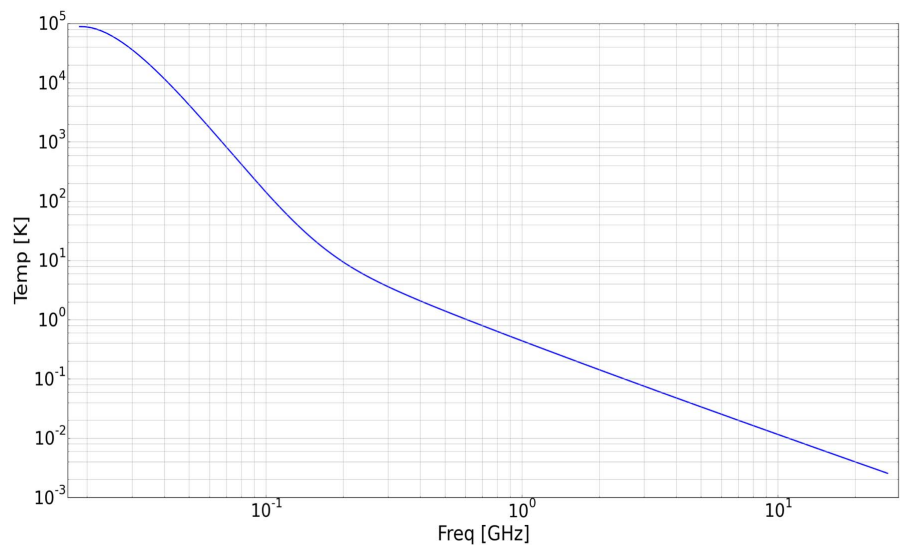

(c) More complex shape that is predominantly concave

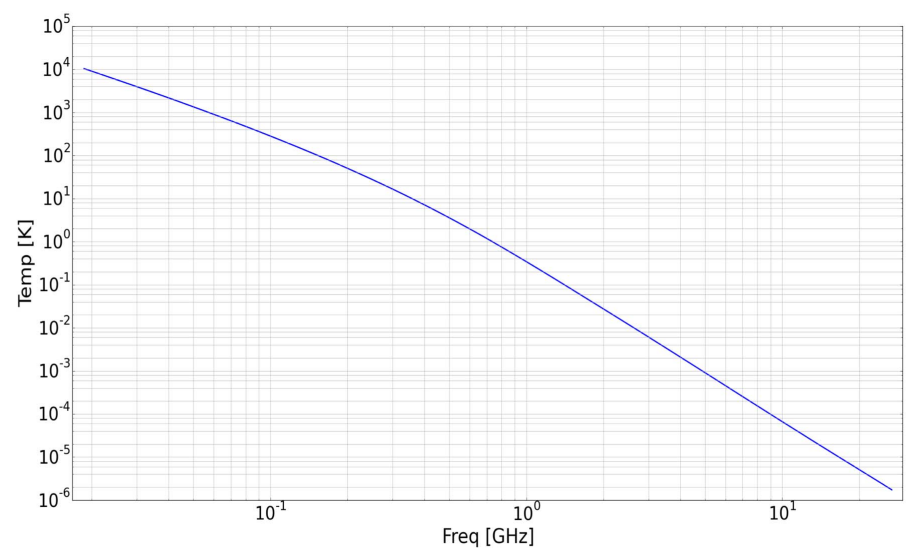

(b) Convex

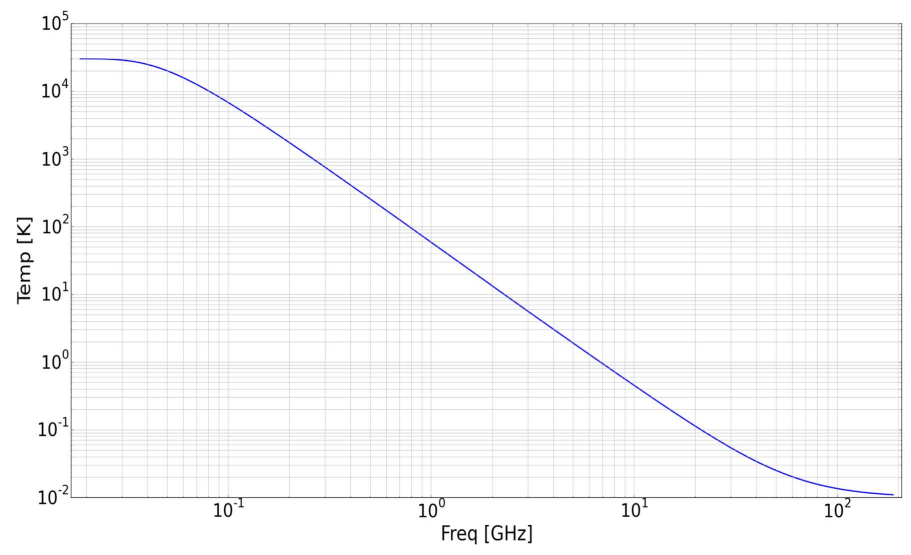

(d) More complex shape that is predominantly convex

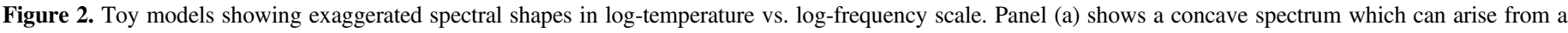

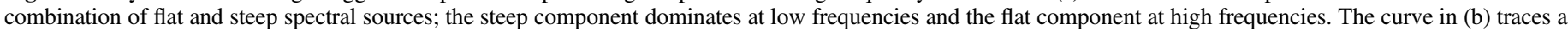

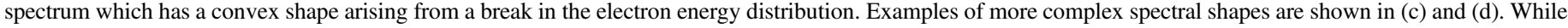

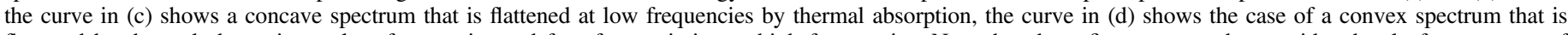

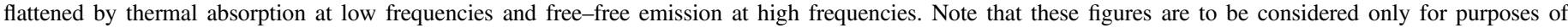
representation of spectral shapes.

here is given by

$$
\begin{aligned}
T(\nu)= & C_{1}\left(\nu^{-\alpha_{1}}+\frac{C_{2}}{C_{1}} \nu^{-\alpha_{2}}+I_{\mathrm{x}} \nu^{-2.1}\right) e^{-\left(\frac{\nu_{\mathrm{t}}}{\nu}\right)^{2.1}} \\
& +T_{\mathrm{e}}\left(1-e^{-\left(\frac{\nu_{\mathrm{t}}}{\nu}\right)^{2.1}}\right) .
\end{aligned}
$$

We drop the parameter $\nu_{\mathrm{br}}$ and instead have a second normalization parameter $C_{2} / C_{1}$, which denotes the ratio of contributions from flat and steep spectrum components. The other parameters are the same as in the case of convex spectra. In both cases there are seven parameters to fit for.

\subsection{GMOSS—Methods}

We employ the downhill simplex algorithm (Nelder \& Mead 1965) to optimize the seven free physical parameters of the convex and concave spectral models in GMOSS by minimizing a goodness of fit $\chi^{2}$ toward every pixel. $\chi^{2}$ is computed to be

$$
\chi^{2}=\frac{1}{N} \sum_{i=1}^{N}\left(\frac{T_{\text {data }}\left(\nu_{\mathrm{i}}\right)-T_{\text {model }}\left(\nu_{\mathrm{i}}\right)}{T_{\text {data }}\left(\nu_{\mathrm{i}}\right)}\right)^{2},
$$

where $T_{\text {data }}$ denotes the image data at frequency $\nu_{\mathrm{i}}, T_{\text {model }}$ denotes the model prediction at the frequency corresponding to the image data, and $N$ is the number of data points available at the sky pixel at which the fit is being done. This constrains the model to fit the measurements with minimal fractional errors, which is appropriate if all the radio images have the same fractional errors. Despite each sky pixel being modeled with a larger number of parameters (7) than data points (6), the model spectrum for any pixel does not exactly fit all the data points for that pixel. The deviations from the data are caused by the defined nature of the model in terms of physically motivated constraints and emission processes that allow only a specific family of curves. The high dimensionality of the modeling problem requires considerable sophistication in the algorithm to obtain a good fit that converges to the global minimum in the multi-dimensional parameter space. Markov chain Monte Carlo (MCMC) is more computationally expensive than the downhill simplex algorithm adopted herein. Future upgrades of GMOSS will adopt an implementation using MCMC as more all-sky radio images become available. The model can accommodate more input maps, including maps with partial sky coverage, 
and can also weight images in a way that is appropriate to their individual fractional errors.

Initial guesses for parameters $\alpha_{1}$ and $\alpha_{2}$ (and hence for $\delta_{\alpha}$ ) are obtained by computing 2-point temperature spectral indices between the data at 45 and $150 \mathrm{MHz}$ and between the data at 408 and $1420 \mathrm{MHz}$, respectively. These determine the model employed to be of either the convex or the concave form. Initial guesses for normalization parameters $C_{1}$ and $C_{2}$ are evaluated at $1420 \mathrm{MHz}$. Initial guesses for parameters $T_{\mathrm{e}}$ and $\nu_{\mathrm{t}}$ are set to the nominal expectations of $8000 \mathrm{~K}$ and $1 \mathrm{MHz}$, respectively. $I_{\mathrm{x}}$ is computed from the difference between the brightness temperature in the data at $23 \mathrm{GHz}$ and that corresponding to the model evaluated after the optically thin high-frequency emission is omitted; however, if the difference is negative, then the initial guess for this parameter is set to be vanishingly small. Further, to aid the optimization toward realistic solutions, certain parameters are constrained within physically acceptable limits. The temperature spectral indices are constrained between 2.0 and 3.0 (Bennett et al. 1992), and the physical temperature $T_{\mathrm{e}}$ for the thermal foreground component that provides the lowfrequency thermal absorption is not allowed to exceed 10,000 K (Haffner et al. 2009).

The synchrotron spectrum requires integration over spectra arising from individual electrons, where a single-electron spectrum itself requires integration of the modified Bessel function of the second kind. The choice of numerical integration technique is critical to accurately and efficiently implementing GMOSS. While an adaptive integration method aids in hastening computing time, care must be taken so that the numerical approximations in the algorithm do not introduce unphysical discontinuities or excessive error in the spectrum owing to computational noise. We use a combination of adaptive integration methods to optimize the speed of computation and numerical accuracy. Adaptive Gauss-Kronrod quadrature is used for all integrals in estimating the parameters, and the open Rhomberg adaptive method is used for integrals that generate the output spectra in specific bands where an accurate representation is desired. For example, for generating foreground spectra for evaluating algorithms for detecting EoR signatures, the open Rhomberg adaptive method is used for integrals that generate the spectrum in a contiguous set of frequencies in the $40-200 \mathrm{MHz}$ band, which is of interest to EoR science. The method of integration allows for a trade-off between speed and accuracy as desired.

The bottleneck in computing time is significantly reduced by using analytic approximations for the integral of the modified Bessel function. A first-order analytical approximation for the integral is given in Rybicki \& Lightman (1986). This is further simplified using the mathematical software tool "Mathematica" (Wolfram Research, Inc. 2016) to reduce the number of integrations by an order of magnitude. For values of $x \geqslant 3$, Equation (2) can be approximated by

$$
\begin{aligned}
F(x)= & \frac{1}{967458816 \sqrt{2} x^{\frac{5}{2}}} e^{-\mathrm{x}}(13 \sqrt{\pi}\{2429625 \\
& \left.+2 x\left(-1922325+5418382 x+83221732 x^{2}\right)\right\} \\
& \left.-1196306216 e^{\mathrm{x}} \pi x^{7 / 2} \operatorname{Erfc}[\sqrt{x}]\right) .
\end{aligned}
$$

Here Erfc is the complementary error function. For $x<3$, Equation (2) is approximated by

$$
\begin{aligned}
F(x)= & -\frac{\pi x}{\sqrt{3}}+\frac{9 x^{11 / 3} \Gamma\left[-\frac{2}{3}\right]}{1602^{2 / 3}} \\
& -\frac{x^{\frac{1}{3}}\left(16+3 x^{2}\right) \Gamma\left[-\frac{1}{3}\right]}{242^{1 / 3}} .
\end{aligned}
$$

Here, $\Gamma(x)$ is the gamma function. Both approximations deviate from the exact treatment by less than $0.1 \%$.

\section{GMOSS PARAMETER VALUES AND GOODNESS OF FIT}

The physical sky model fits the data at all 3072 pixels with a median $\chi^{2}$ of 0.0034 , corresponding to a mean fractional departure of $6 \%$. Ninety-nine percent of the pixels have $\chi^{2}$ less than 0.03 , corresponding to a mean fractional error of $17 \%$; a histogram of $\chi^{2}$ is shown in Figure 3. Also shown in Figure 3 is an all-sky map of $\chi^{2}$ on a Mollweide projection of the sky in Galactic coordinates. Surprisingly, the errors are relatively smaller where the Galaxy dominates, both on the plane and in the region of the north polar spur. In these regions the model corresponding to a convex spectrum was selected by the fitting algorithm.

Shown in Figure 4 are sample spectra derived from GMOSS (blue solid lines) toward three pixels that are representative of convex (pixel 36), concave (pixel 2060), and more complex (pixel 1130) spectral shapes. The measurement data from the six maps are also shown in the three panels using filled red circles. Figure 4 also shows in a panel the locations of these pixels as an image in Galactic coordinates. The chosen pixels lie toward different sky regions, where the spectral shapes are dominated by emission from different components. As demonstrated by the fit solution to sample pixel 1130, not only is the emission from the Galactic center region brighter, as expected, than emission away from the plane, but the spectral structure is also more complex, necessitating modeling with a broken power-law synchrotron emission plus significant lowfrequency turnover due to thermal absorption and excess freefree emission at the high-frequency end. This is to be expected from the variety of components and processes that are unique to the Galactic plane, particularly the Galactic center, including $\mathrm{H}$ II regions and supernova remnants, to name a few. Pixel 36 is in the vicinity of the central bulge, and the fit solution is that of a convex spectrum in which the emission is modeled in GMOSS as a broken power-law synchrotron spectrum, without significant thermal effects needed, which are mainly present toward the Galactic plane. The spectrum at pixel 2060 is in the vicinity of the Galactic pole and has a concave form. The spectrum toward this pixel is modeled by GMOSS as composite emission from flat and steep spectrum components.

Two sample all-sky maps at 50 and $200 \mathrm{MHz}$ generated using GMOSS are shown in Figure 5. The Mollweide projection maps are in Galactic coordinates with $5^{\circ}$ resolution. With this resolution, coarse Galactic features clearly arise in the maps, and the mean temperatures are higher in the lowerfrequency map as expected. These maps have a median deviation from their nearest input maps-namely, the ones at $45 \mathrm{MHz}$ and $150 \mathrm{MHz}$ - by $25 \%$ and $50 \%$, respectively.

The panels in Figure 6 show the distribution of optimized parameters across the 3072 sky pixels on Mollweide 


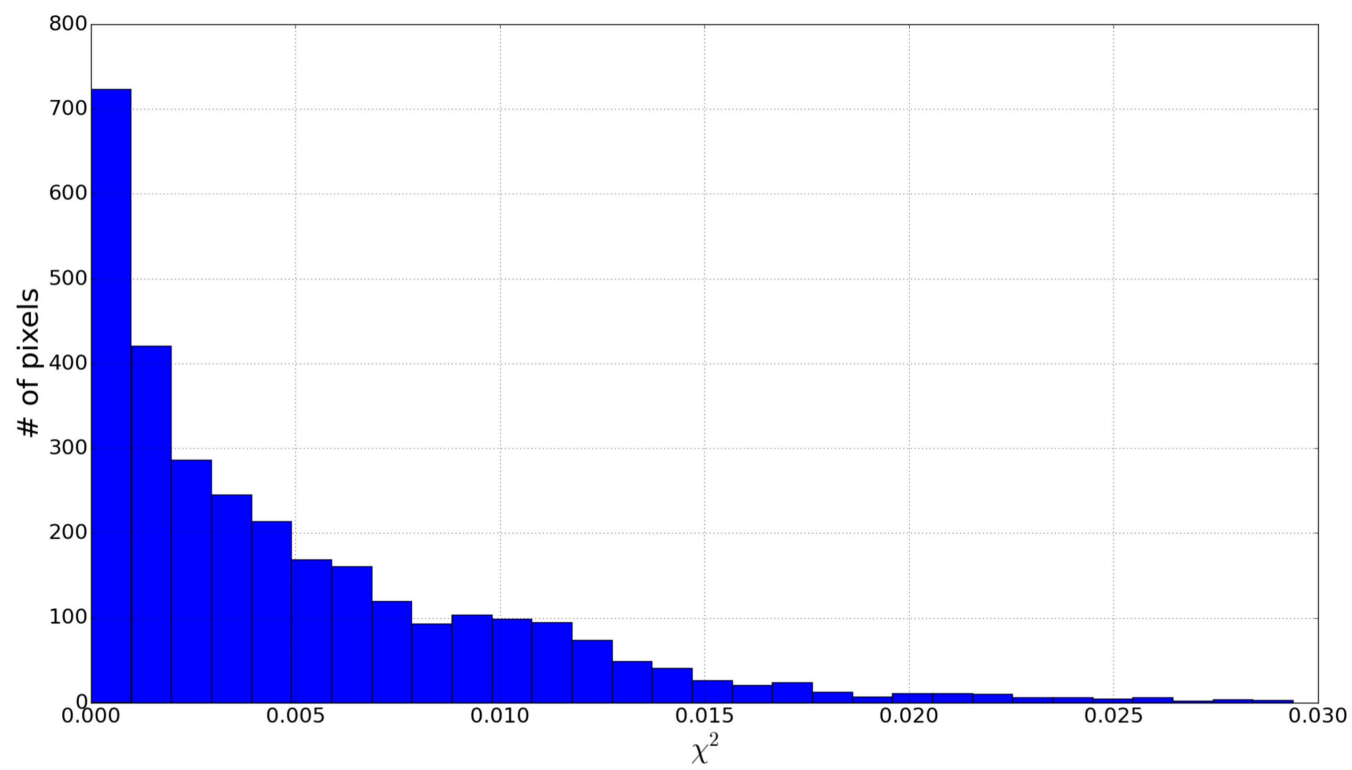

(a)
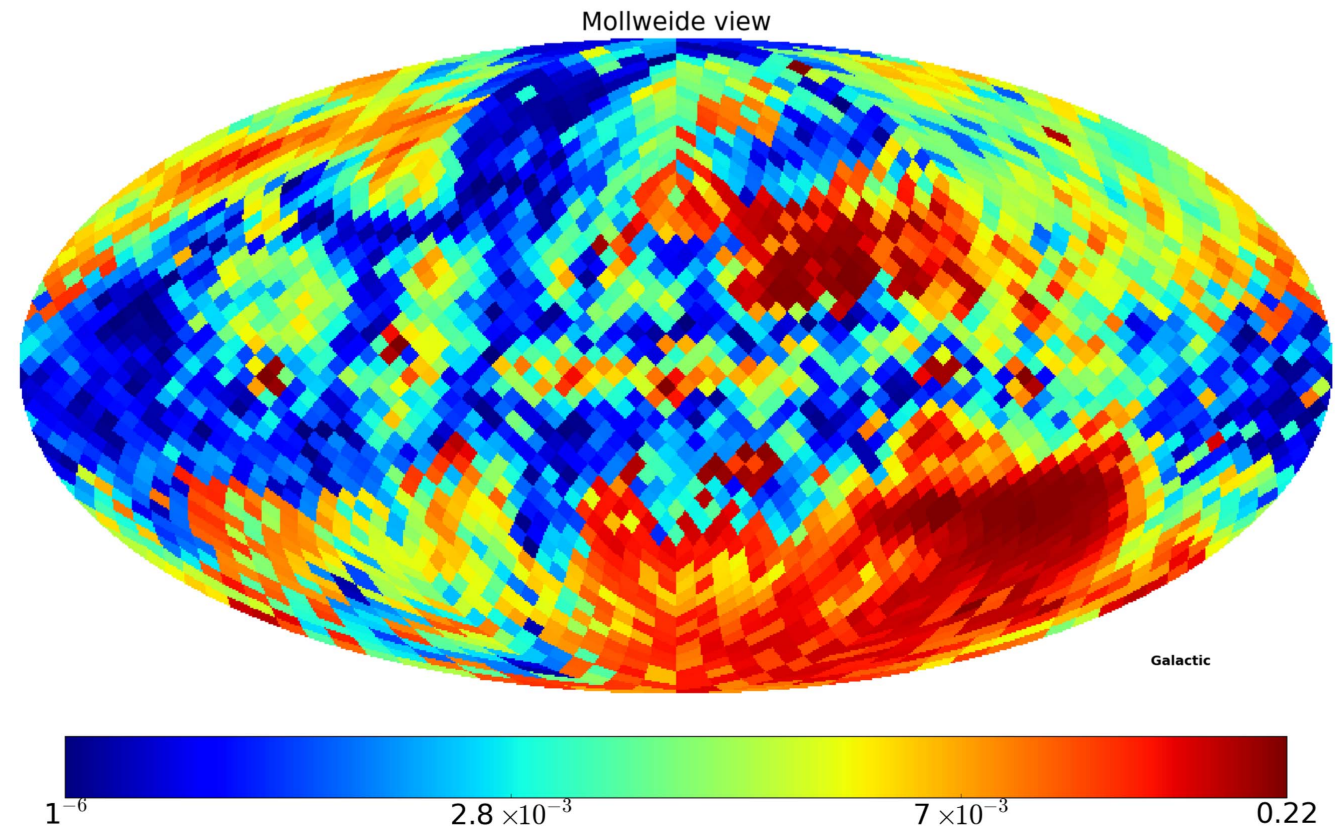

(b)

Figure 3. (a) Histogram of goodness of fit $\chi^{2}$ computed for all 3072 pixels. The median of the distribution is 0.0034 , the mean is 0.0054 , and the maximum value is 0.2280. (b) The distribution of the $\chi^{2}$ values computed over the 3072 sky pixels is shown in a Mollweide projection, in Galactic coordinates with $5^{\circ}$ resolution.

projections of the sky in Galactic coordinates. Panels showing the break frequency and secondary normalization parameters in convex and concave spectra, respectively, are mutually exclusive in modeling the sky spectrum in GMOSS. In each case, pixels that have spectra of the other form are masked and are in gray. The median values of some of the optimized parameters are given in Table 1.

Figure 6(a) shows the relative distributions of pixels that have convex and concave spectra and thus entail two different models for the relativistic electrons. It is interesting to note that these pixels employing different models are distributed toward distinct regions of the sky. The Galactic plane and the north polar spur are distinct and require convex spectra employing a break in the power-law synchrotron emission, with the spectral fit toward pixel 36 in Figure 4 serving as a representative example. In contrast, the radio spectra in regions off the Galactic plane and the spur are concave and hence required modeling as a composite of flat and steep spectrum components; the spectral fit toward pixel 2060 in Figure 4 is a representative example of this type.

The above difference is likely a reflection of the difference in origin of relativistic electron populations. In the Galaxy, electrons are believed to be created in shock accelerations associated with supernovae, and these then diffuse and migrate off the plane, acquiring a break in the electron energy distribution because of aging and loss mechanisms (Lisenfeld 


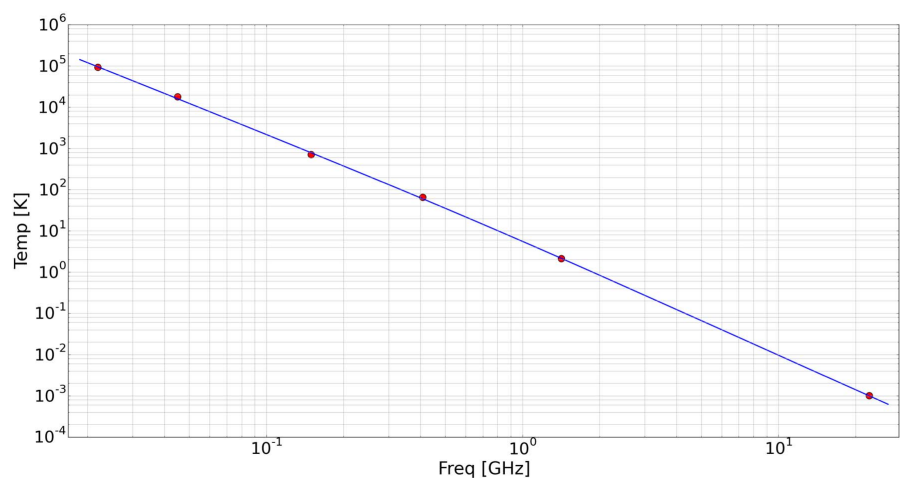

(a)

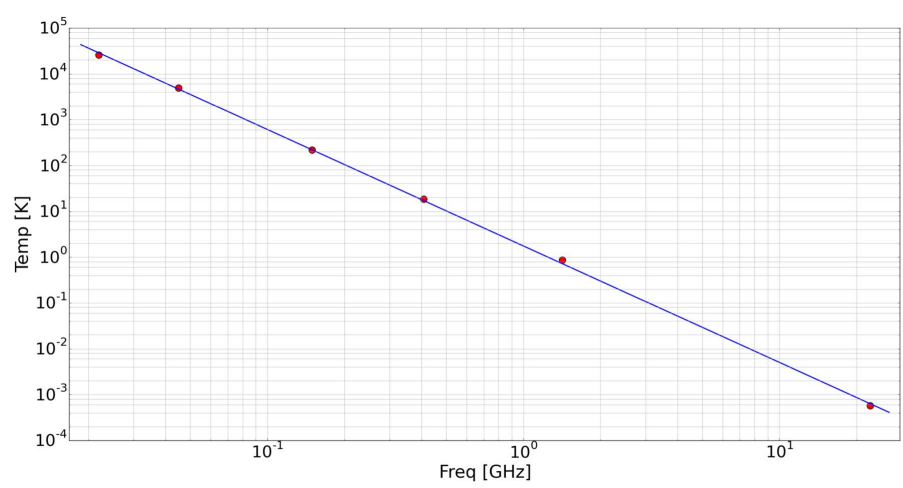

(b)

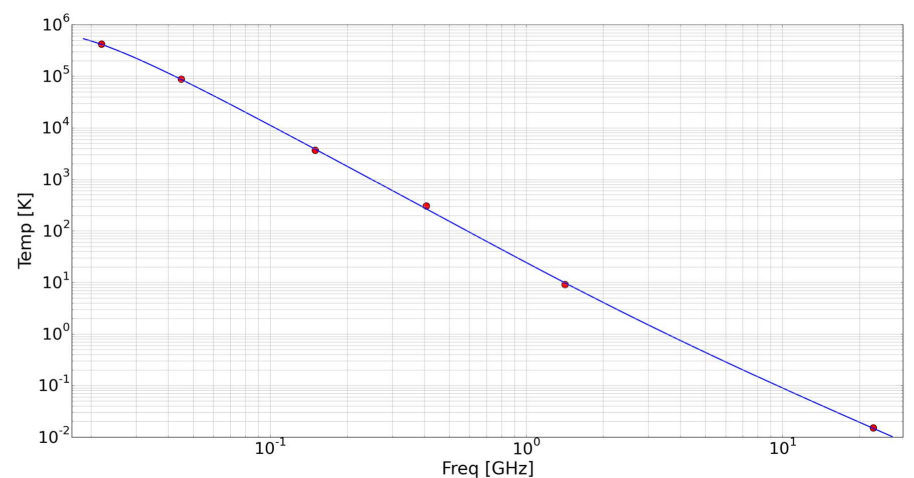

(c)

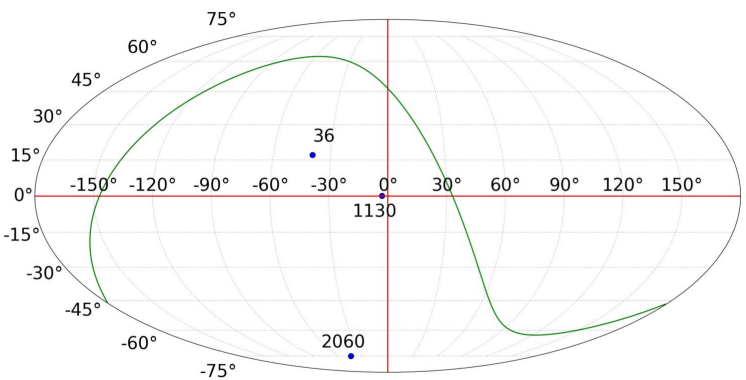

(d)

Figure 4. Data points (filled red circles) toward representative pixels with overlaid GMOSS-generated spectra (solid blue lines). The pixel positions selected for display contain (a) a convex shape at pixel 36, (b) a concave shape at pixel 2060, and (c) a complex shape at pixel 1130. The plots are on a log-temperature vs. logfrequency scale. Panel (d) shows the positions of these pixels on a Mollweide projection of the sky in Galactic coordinates, where the solid green line traces the ecliptic.

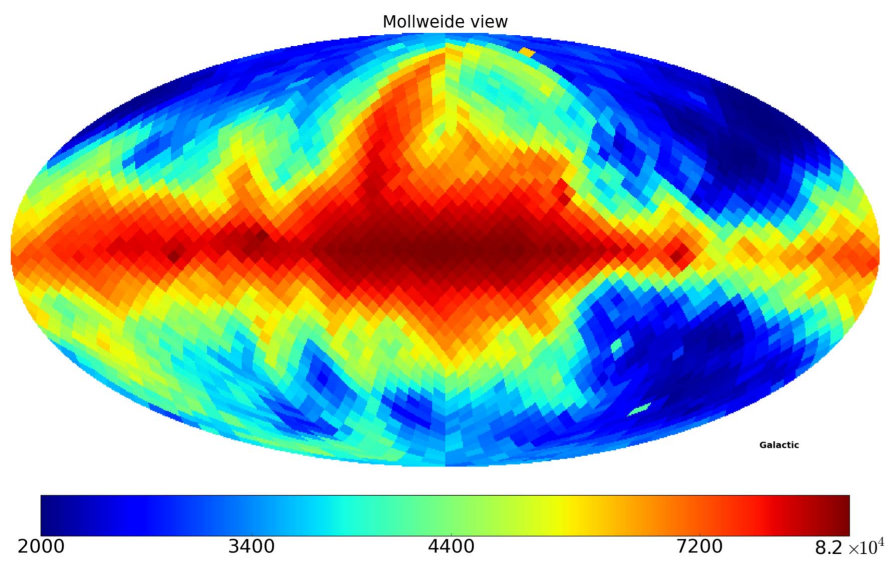

(a)

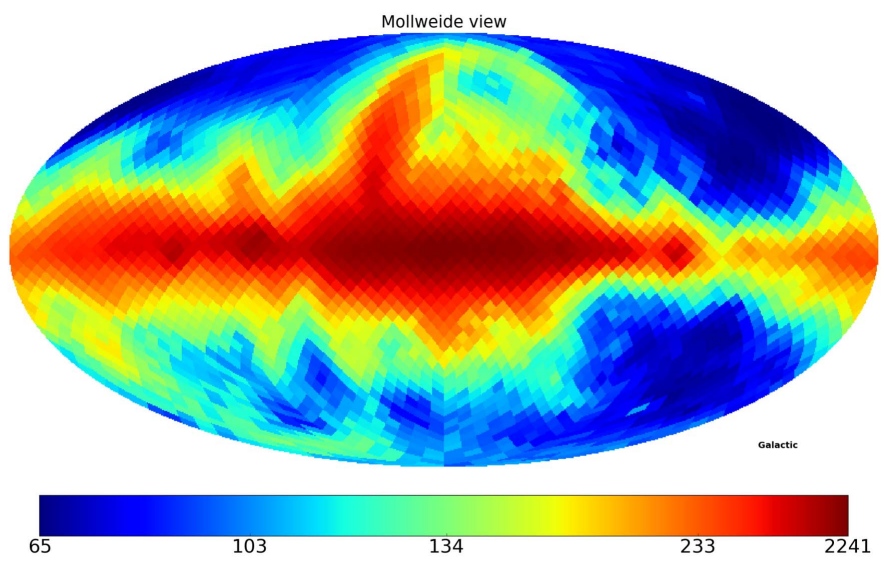

(b)

Figure 5. All-sky maps derived from GMS at (a) $50 \mathrm{MHz}$ and (b) $200 \mathrm{MHz}$. The maps are in units of kelvin at a resolution of $5^{\circ}$ and in Galactic coordinates.

\& Völk 2000). On the other hand, extragalactic radio emission is dominated at low frequencies by powerful radio galaxies in which acceleration is in hot spots at the ends of relativistic jets and perhaps in situ re-acceleration is in cocoons. At high frequencies, the dominant emission is from flat spectrum cores of active galactic nuclei (see, for example, Miley 1980). This consistency between GMOSS results and expectations lends confidence to the modeling presented here.
Figure 6(b) gives the distribution of the temperature spectral index $\alpha_{1}$ of the low-frequency synchrotron emission across the sky. The parameter $\delta_{\alpha}$ that represents the change in spectral index toward high frequencies is shown in Figure 6(c). Unsurprisingly, pixels that have positive values of $\delta_{\alpha}$ have convex spectra, represented in black in Figure 6(a), and those with negative values have spectra of the concave shape, given as white pixels in Figure 6(a). 


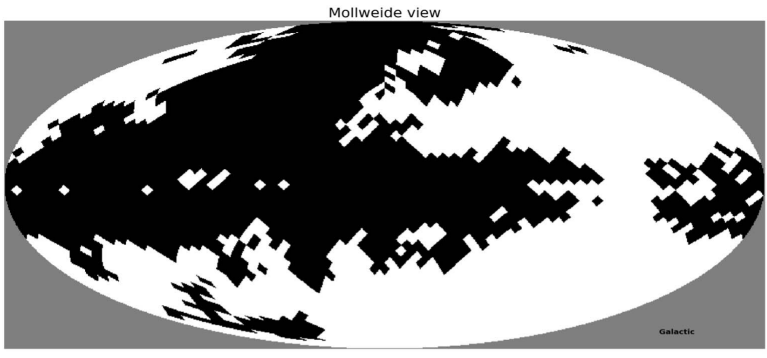

(a)

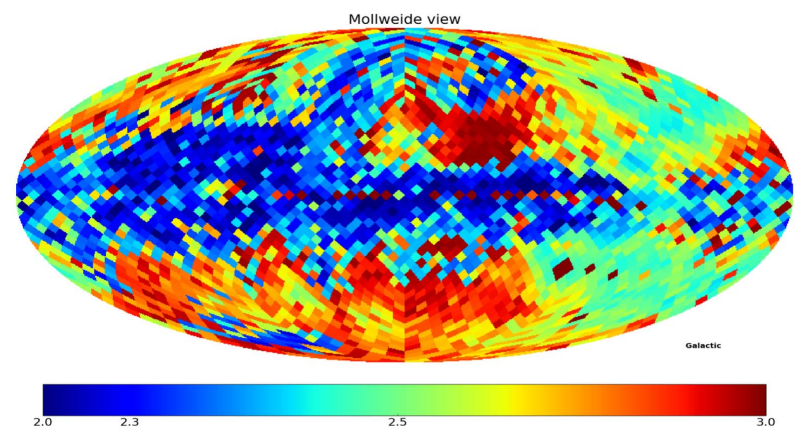

(b)

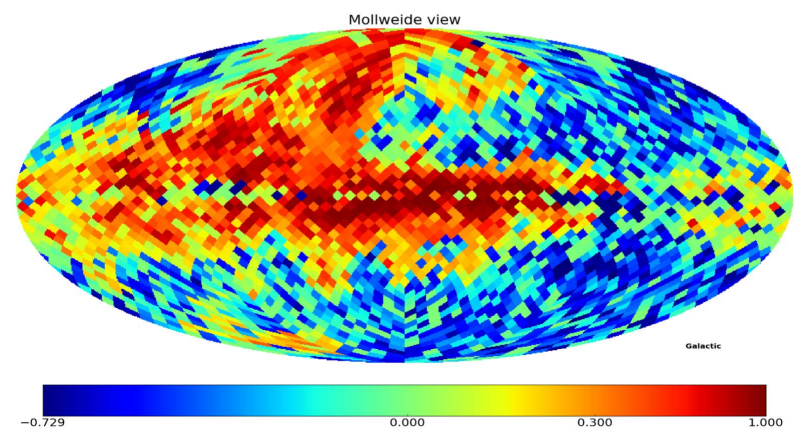

(c)

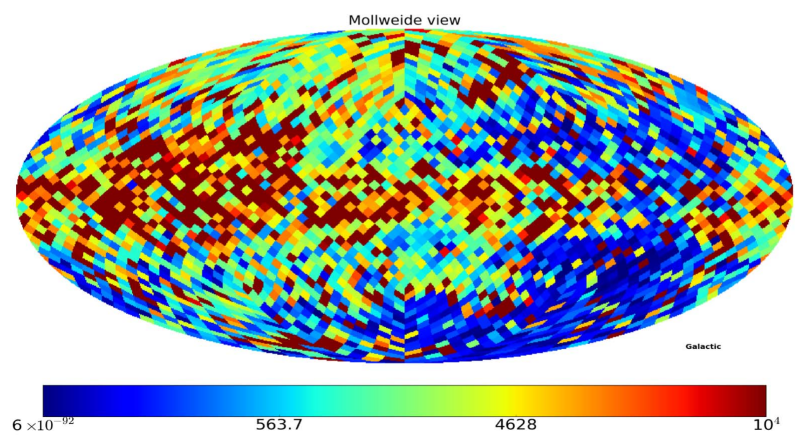

(d)

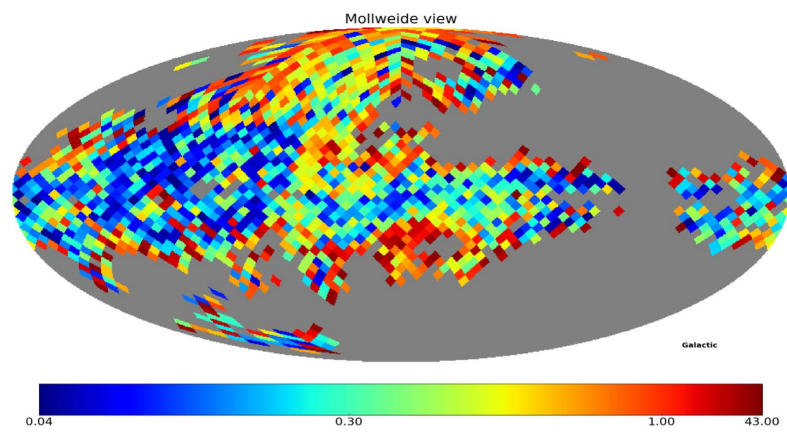

(e)

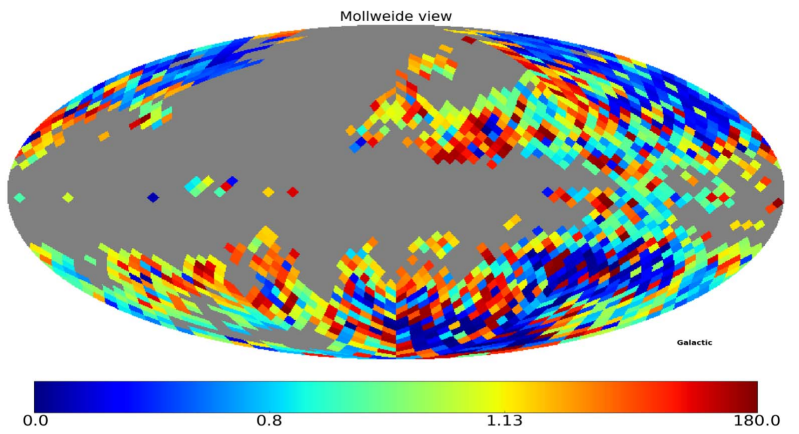

(f)

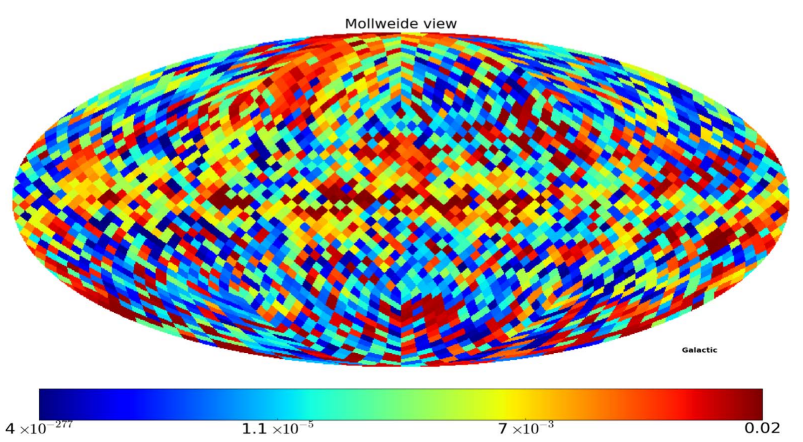

(g)

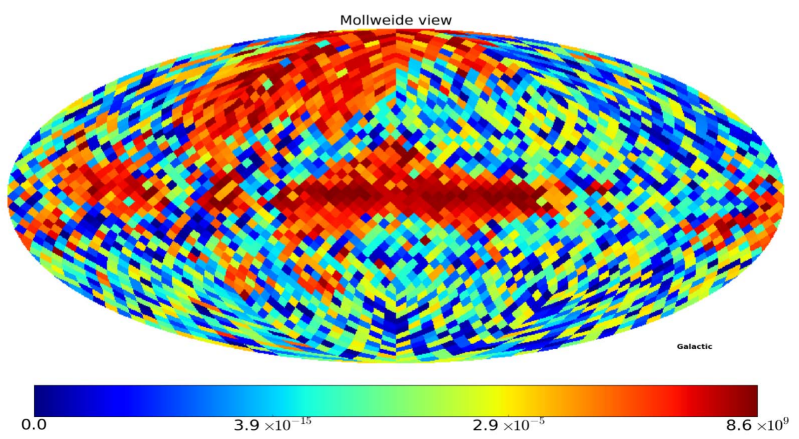

(h)

Figure 6. Sky distribution of the optimized values of the parameters. All maps are at a resolution of $5^{\circ}$ and in galactic coordinates. (a) Distribution of pixels with convex (black) and concave (white) spectra. The Galactic plane and north polar spur are clearly of distinct types, suggesting different physics in the electron populations compared to those of the extragalactic sky. (b) Shows $\alpha_{1}$. (c) $\delta_{\alpha}$ is given as positive when $\alpha_{2}>\alpha_{1}$ and as negative when $\alpha_{2}<\alpha_{1}$, such that $\alpha_{2}=\alpha_{1}+\delta_{\alpha}$. (d) Temperature $T_{\mathrm{e}}$ and (e) break frequency $\nu_{\mathrm{br}}$ for convex spectra in GHz. (f) The additional normalization for the flat spectrum sources, $C_{2}$, for concave spectra; (g) the frequency of thermal absorption turnover, $\nu_{\mathrm{t}}$; and $(\mathrm{h})$ the parameter representing optically thin free-free emission, $I_{\mathrm{x}}$. 
Table 1

Median Values of Optimized Parameters Over All 3072 Pixels That Describe the Sky Models

\begin{tabular}{lc}
\hline \hline Parameter & Median Value \\
\hline$\alpha_{1}$ & 2.50 \\
$\alpha_{2}$ & 2.58 \\
$\nu_{\mathrm{br}}$ & $0.36 \mathrm{GHz}$ \\
$I_{\mathrm{x}}$ & $8.39 \times 10^{-10}$ \\
$T_{\mathrm{e}}$ & $2060 \mathrm{~K}$ \\
$\nu_{\mathrm{t}}$ & $0.3 \mathrm{MHz}$ \\
\hline
\end{tabular}

The temperature of the thermal medium that models the absorption at low frequencies is shown in Figure 6(d), and the frequency of the thermal absorption turnover is in Figure $6(\mathrm{~g})$. Pixels with the highest temperatures and the highest turnover frequencies lie mostly along the Galactic plane and toward the north polar spur. Pixels in blue in Figure 6(g) have very low values, indicating that the extragalactic sky does not require any significant thermal absorption in the physical modeling. The median thermal absorption turnover frequency, $\nu_{\mathrm{t}}$, in the GMOSS modeling is $0.3 \mathrm{MHz}$, which is consistent with estimates in literature (Novaco \& Brown 1978; Cane 1979) that this value is in the ballpark of $1 \mathrm{MHz}$. The median value of the GMOSS parameter $T_{\mathrm{e}}$ for the ionized medium that models this turnover is $2060 \mathrm{~K}$, which is in the range of electron temperatures estimated for the interstellar medium: from observations of radio recombination lines (RRLs) at 328, 75, and $34.5 \mathrm{MHz}$ Kantharia \& Anantharamaiah (2001) estimate the extended low-density warm ionized medium (ELDWIM) responsible for carbon recombination lines to have $T_{\mathrm{e}}$ as low as 30-300 K, and based on $1.4 \mathrm{GHz}$ recombination lines, Heiles et al. (1996) estimate the ELDWIM to have $T_{\mathrm{e}}$ of $7000 \mathrm{~K}$.

Figures 6(e) and (f) represent two mutually exclusive parameters of the physical models in GMOSS-namely, the break frequency $\nu_{\mathrm{br}}$ for pixels that have convex spectra, and a secondary normalization $C_{2}$ for the flat-spectrum emission component in the case of pixels that have concave spectra. The median value of $\nu_{\mathrm{br}}$ is $360 \mathrm{MHz}$ (see Table 1). This is consistent with the pure diffusion model of Galactic cosmic ray electron propagation in Strong et al. (2011) for a break in the electron injection spectrum of $4.0 \mathrm{GeV}$, if the magnetic field is $1.4 \mu \mathrm{G}$.

Lastly, Figure 6(h) shows the parameter $I_{\mathrm{x}}$, which represents optically thin free-free emission that dominates at high frequencies. This parameter clearly arises from a component that lies along the north polar spur and a region of the Galactic plane with a relatively smaller scale-height. The pixels with spectra showing spectral steepening as well as significant optically thin free-free emission would most certainly have a spectral shape that is complex, due to the convex spectrum at low frequencies followed by an upturn at high frequencies, where excess emission dominates; the spectrum toward pixel 1130, as shown in Figure 4, is an example of this type.

Using GMOSS-generated model spectra at each pixel, we have computed the 2-point spectral indices for the pair of frequencies 50 and $150 \mathrm{MHz}$ and separately for the pair 400 and $1200 \mathrm{MHz}$. Histograms of these 2-point spectral indices are shown in Figure 7. The computed spectral indices have a mean value of 2.49 at the lower set of frequencies, where the spectral indices are distributed over the range 2.27-2.76. Within the errors, this is the same as the median value of 2.50 for the $\alpha_{1}$ model parameter (listed in Table 1), which represents the spectral index of the synchrotron component below any break. This low-frequency spectral index is also consistent with the observations of Rogers \& Bowman (2008). At relatively higher frequencies, the 2-point spectral index between 400 and $1200 \mathrm{MHz}$ is 2.56 , and in this band the range is over 2.24-2.85. The median value of the spectral index parameter $\alpha_{2}$ above any break is 2.58 and is in the same ballpark as the median 2-point spectral index. The high-frequency spectral index is again in agreement with measurements of the absolute radio sky at $\mathrm{cm}$ wavelengths (see, for example, Kogut et al. 2011).

\section{SUMMARY}

We have presented a physically motivated sky model of the low-frequency radio sky from $22 \mathrm{MHz}$ to $23 \mathrm{GHz}$. Spectra over this frequency range are presented over 3072 pixels covering the sky in R4 HEALPix pixels; the resolution is $5^{\circ}$. GMOSS models the spectrum at each pixel primarily as optically thin synchrotron emission, adopting either electron distribution of a broken power-law form or a composite of steep and flat spectrum components. Additionally, optically thin thermal emission is included to correct for any deficit at the high-frequency end, and thermal absorption is added as a foreground screen to account for any low-frequency flattening. The 7-parameter model is fit to six all-sky images at $0.022,0.045,0.150,0.408,1.420$, and $23 \mathrm{GHz}$ to derive the GMOSS model spectra. The fractional differences between the GMOSS model spectra and the input data have a median value of $6 \%$. This is in keeping with the systematic calibration errors in the input maps, which range from $1 \%$ to $20 \%$. Furthermore, derived physical parameters of the model are in reasonable agreement with expected values, providing confidence in the physical model.

With a resolution of $5^{\circ}$, which is much finer than that of typical antenna beams used in experiments attempting to detect the global signature of EoR, the model can be convolved with appropriate antenna beams in simulations of these experiments to generate mock sky spectra. GMOSS provides an expectation of the EoR foreground to help one arrive at appropriate component separation strategies without any inherent assumptions of smoothness in the foreground spectrum. It may be noted here that GMOSS is intended to represent the radio continuum emission, and the physical processes included are those relevant to the radio continuum in the $\mathrm{MHz}-$ to- $\mathrm{GHz}$ frequency range. This is useful for modeling the foreground contamination of wideband cosmological spectral signals or distortions of the CMB spectrum in these wavelengths. RRLs from the Galactic thermal component would be expected to add sharp spectral features and would hence be distinguishable from wideband spectral signals, such as those expected from the recombination and reionization epochs and the Dark Ages and Cosmic Dawn in between. Hence, RRLs are not included in GMOSS.

Also, it may be noted that the all-sky radio maps used as inputs to GMOSS - and indeed all-sky maps that may be available in the foreseeable future-do not have accuracy anywhere near the level of the cosmological signals mentioned above. Including more images at intermediate frequencies in the coming years, as new images become available, will most certainly improve GMOSS. If the fits yield residuals outside the error bars of the images, then more spectral complexity would necessarily have to be added to the model. However, allowing for multiple breaks (which are indeed observed in sources 


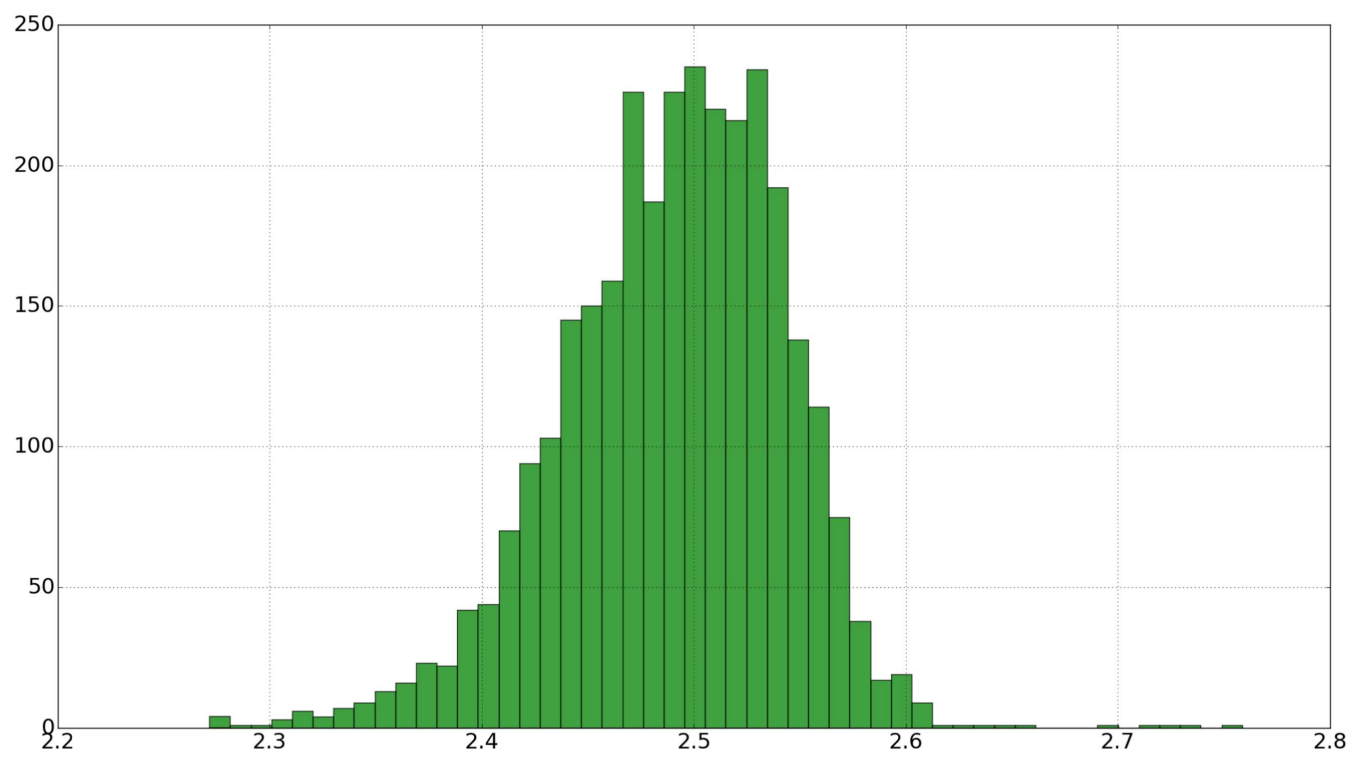

(a)

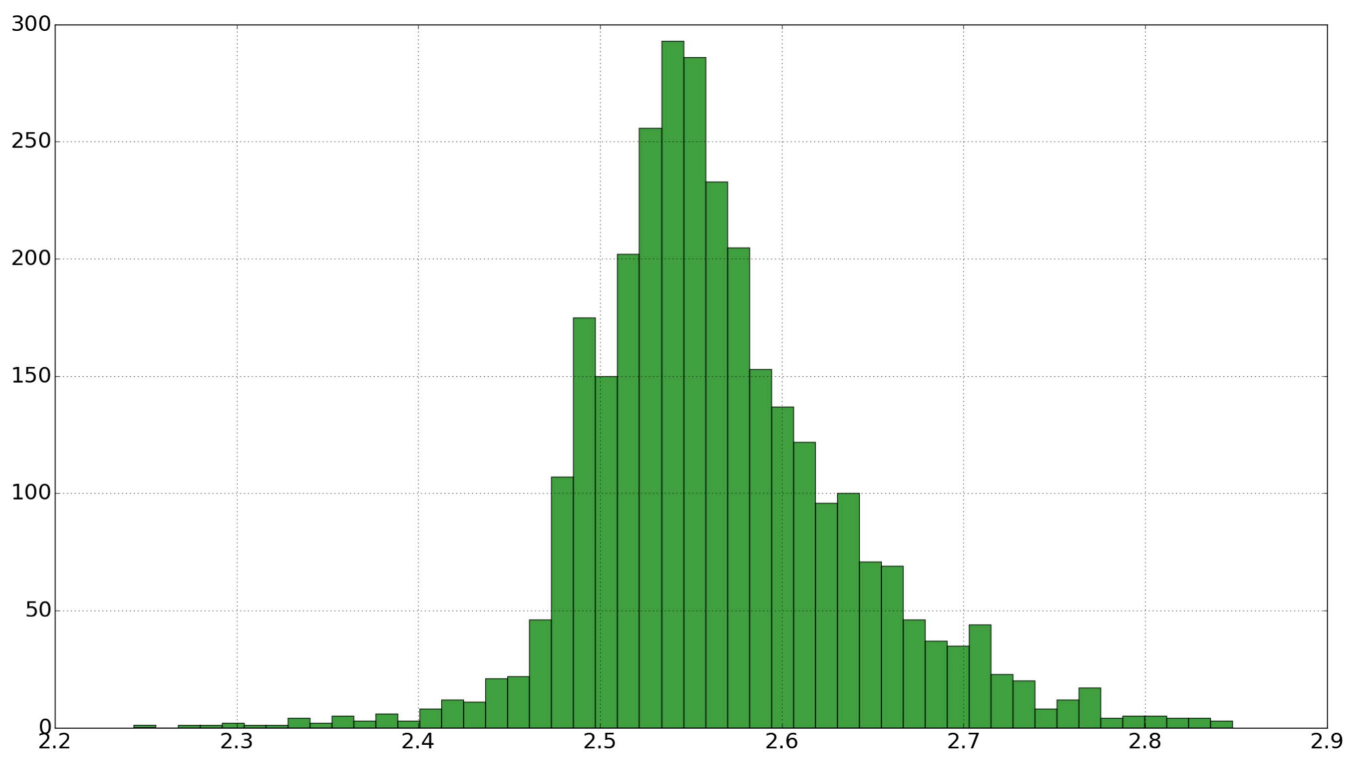

(b)

Figure 7. Histogram of 2-point spectral indices derived from GMOSS at all 3072 pixels (a) between 50 and $150 \mathrm{MHz}$ and (b) between 400 and $1200 \mathrm{MHz}$.

where multiple cooling processes are presumed to occur) and/ or multiple spectral components would most likely satisfy improved data without requiring additional radiative processes. It is not impossible that such inclusions might result in increased complexity of the synthetic spectra and hence prove to cause greater confusion to the detection of cosmological signals.

A study of the implications of GMOSS-predicted sky spectra for EoR signal detection and signal extraction strategies is presented in a subsequent paper (Sathyanarayana Rao et al. 2016, in preparation). This subsequent paper presents among other things a comparison of GMOSS with existing polynomial models for foregrounds as well as a discussion on regions of the sky best suited for EoR signal detection experiments.

However, since GMOSS is not tailored to EoR science but based entirely on plausible physics that produce the radio sky spectrum, it may be used for any problem that requires simulating the radio sky spectrum. Simulation studies of foreground contamination in $\mathrm{cm}$-wavelength detection experiments of signals arising from the epoch of recombination are one such area where GMOSS can be applied. GMOSS is publicly available at ${ }^{6}$ www.rri.res.in/DISTORTION/ and will be updated as other maps and processes are included. The code used to generate the model is flexible and can include more maps, including those with partial sky coverage.

We acknowledge the use of the Legacy Archive for Microwave Background Data Analysis (LAMBDA), part of the High Energy Astrophysics Science Archive Center (HEASARC). HEASARC/LAMBDA is a service of the

\footnotetext{
6 Also available at https://github.com/RRIDISTORTION/GMOSS
} 
Astrophysics Science Division at the NASA Goddard Space Flight Center. Some of the results in this paper have been derived using the HEALPix (Górski et al. 2005) package. M.S.R. would like to thank K. S. Dwarakanath for their useful discussions on Galactic radio emission. J.C. is supported by the Royal Society as a Royal Society University Research Fellow at the University of Manchester, U.K.

\section{REFERENCES}

Ali, Z. S., Parsons, A. R., Zheng, H., et al. 2015, ApJ, 809, 61 Bennett, C. L., Smoot, G. F., Hinshaw, G., et al. 1992, ApJL, 396, L7 Bernardi, G., McQuinn, M., \& Greenhill, L. J. 2015, ApJ, 799, 90

Bowman, J. D., Rogers, A. E. E., \& Hewitt, J. N. 2008, ApJ, 676, 1 Cane, H. V. 1979, MNRAS, 189, 465

de Oliveira-Costa, A., Tegmark, M., Gaensler, B. M., et al. 2008, MNRAS, 388,247

Furlanetto, S. R., Oh, S. P., \& Briggs, F. H. 2006, PhR, 433, 181

Glover, S. C. O., Chluba, J., Furlanetto, S. R., Pritchard, J. R., \& Savin, D. W. 2014, AAMOP, 63, 135

Górski, K. M., Hivon, E., Banday, A. J., et al. 2005, ApJ, 622, 759

Haffner, L. M., Dettmar, R.-J., Beckman, J. E., et al. 2009, RvMP, 81, 969

Harker, G. J. A. 2015, MNRAS, 449, L21

Harker, G. J. A., Mirocha, J., Burns, J. O., \& Pritchard, J. R. 2016, MNRAS, 455,3829

Haslam, C. G. T., Salter, C. J., Stoffel, H., \& Wilson, W. E. 1982, A\&AS, 47, 1 Heiles, C., Reach, W. T., \& Koo, B.-C. 1996, ApJ, 466, 191

Kantharia, N. G., \& Anantharamaiah, K. R. 2001, JApA, 22, 51

Kogut, A., Fixsen, D. J., Levin, S. M., et al. 2011, ApJ, 734, 4

Landecker, T. L., \& Wielebinski, R. 1970, AuJPA, 16, 1
Lisenfeld, U., \& Völk, H. J. 2000, A\&A, 354, 423

Madau, P., Meiksin, A., \& Rees, M. J. 1997, ApJ, 475, 429

Miley, G. 1980, ARA\&A, 18, 165

Nelder, J. A., \& Mead, R. 1965, CompJ, 7, 308

Novaco, J. C., \& Brown, L. W. 1978, ApJ, 221, 114

Patra, N., Subrahmanyan, R., Raghunathan, A., \& Udaya Shankar, N. 2013, ExA, 36, 319

Patra, N., Subrahmanyan, R., Sethi, S., Udaya Shankar, N., \& Raghunathan, A. 2015, ApJ, 801, 138

Petrovic, N., \& Oh, S. P. 2011, MNRAS, 413, 2103

Pober, J. C., Ali, Z. S., Parsons, A. R., et al. 2015, ApJ, 809, 62

Pober, J. C., Liu, A., Dillon, J. S., et al. 2014, ApJ, 782, 66

Pritchard, J., \& Loeb, A. 2010a, Natur, 468, 772

Pritchard, J. R., \& Loeb, A. 2010b, PhRvD, 82, 023006

Reich, P., \& Reich, W. 1986, A\&AS, 63, 205

Reich, W. 1982, A\&AS, 48, 219

Rogers, A. E. E., \& Bowman, J. D. 2008, AJ, 136, 641

Rybicki, G. B., \& Lightman, A. P. 1986, Radiative Processes in Astrophysics (New York: Wiley)

Sathyanarayana Rao, M., Subrahmanyan, R., Udaya Shankar, N., \& Chluba, J. 2015, ApJ, 810, 3

Shaver, P. A., Windhorst, R. A., Madau, P., \& de Bruyn, A. G. 1999, A\&A, 345,380

Strong, A. W., Orlando, E., \& Jaffe, T. R. 2011, A\&A, 534, A54

Sunyaev, R. A., \& Chluba, J. 2009, AN, 330, 657

Switzer, E. R., \& Liu, A. 2014, ApJ, 793, 102

Voytek, T. C., Natarajan, A., Jáuregui García, J. M., Peterson, J. B., \& López-Cruz, O. 2014, ApJL, 782, L9

Waelkens, A., Jaffe, T., Reinecke, M., Kitaura, F. S., \& Enßlin, T. A. 2009, A\&A, 495, 697

Wolfram Research, Inc. 2016, Mathematica, Version 10.4

Zheng, H., Tegmark, M., Dillon, J., et al. 2016, arXiv:1605.04920 